\title{
The promise of glycoproteomics for studying cardiovascular disease
}

\author{
Abstract \\ The potential of glycoproteomics for analyzing proteins associated with cardiovascular \\ diseases are discussed.
}

Keywords: glycoproteomics, extracellular matrix, myocardial infarction

\author{
Volume I Issue 3 - 2014 \\ Merry L Lindsey, ${ }^{1,2,3}$ Yuan Tian ${ }^{1,2}$ \\ 'Department of Physiology and Biophysics, University of \\ Mississippi Medical Center, USA \\ ${ }^{2}$ San Antonio Cardiovascular Proteomics Center, USA \\ ${ }^{3}$ Research Service, G.V. (Sonny) Montgomery Veterans Affairs \\ Medical Center, USA
}
Correspondence: Yuan Tian, Department of Physiology and Biophysics, University of Mississippi Medical Center, 2500 North State St. Jackson, MS 39216-4505, USA, Tel 6019844795, Fax 6019841833,Email ytian@umc.edu

Received: June 26, 2014 | Published: July 0I, 2014

\section{Editorial}

Protein glycosylation, an enzyme-directed site-specific process, is one of the most common co-translational and post-translational modifications. ${ }^{1}$ Glycoproteins modulate multiple biological processes, including cell adhesion and migration, signal transduction, and cell-cell communication., ${ }^{2,3}$ Despite its widespread importance, glycoproteomics is not commonly used for studying cardiovascular disease compared to other diseases, such as cancers and diabetes. Glycoproteomics has the potential to be a powerful tool for analyzing proteins associated with cardiovascular diseases, as discussed below.

i. Glycosylation alters protein function by influencing protein folding, activity, stability, and distribution. ${ }^{4}$ Glycosylation is increasingly recognized for its importance in modulating cardiomyocyte function and survival. ${ }^{5}$

ii. Glycoproteins are the major components of the cardiac extracellular matrix, including structural and non-structural proteins that play key roles in cardiovascular disease development. ${ }^{6}$ For example, thrombospondin, tenascin-C, and periostin are 3 nonstructural extracellular matrix glycoproteins that modulate cardiac remodeling after myocardial infarction. ${ }^{7-9}$

iii. Since most cell surface and secreted proteins, including extracellular matrix proteins, are glycosylated, glycoproteomics is a useful enrichment strategy for the study of extracellular proteins. ${ }^{10-12}$ Due to the extracellular location, these proteins are readily detected on cell surface or released into circulation, allowing them to serve as potential biomarkers and logical drug targets. ${ }^{13}$ Therefore, glycoproteomics is a good approach for biomarker discovery.

iv. Glycoproteomics greatly reduces the sample complexity by focusing on glycosylated peptides instead of all protein peptides, which greatly improves the odds of the detecting low abundant proteins. ${ }^{14,15}$ Glycoproteome enrichment coupled with targeted mass spectrometry analysis, such as selected reaction monitoring (SRM), further improves the sensitivity of mass spectrometry-based assays. ${ }^{16}$
Glycosylation is a highly abundant modification crucial for the regulation of protein function, including proteolytic cleavage by enzymes and intra-protein interaction. Glycoproteomics is a logical approach to target specific subproteome with improved sensitivity for low abundant proteins. Therefore, glycoproteomics presents a new direction in methods that allow proteins associated with cardiovascular disease to be assessed for potential use as biomarkers or drug targets.

\section{Acknowledgements}

None.

\section{Conflict of interest}

The author declares no conflict of interest.

\section{References}

1. Furukawa K, Kobata A. Protein glycosylation. Curr Opin Biotechnol. 1992;3(5):554-559.

2. Isaji T, Gu J, Nishiuchi R, et al. Introduction of bisecting GlcNAc into integrin alpha5beta1 reduces ligand binding and down-regulates cell adhesion and cell migration. J Biol Chem. 2004;279(19):19747-19754.

3. Rudd PM, Elliott T, Cresswell P, et al. Glycosylation and the immune system. Science. 2001;291(5512):2370-2376.

4. Spiro RG. Protein glycosylation: nature, distribution, enzymatic formation, and disease implications of glycopeptide bonds. Glycobiology. 2002;12(4):43R-56R.

5. Parker BL, Palmisano G, Edwards AV, et al. Quantitative N-linked glycoproteomics of myocardial ischemia and reperfusion injury reveals early remodeling in the extracellular environment. Mol Cell Proteomics. 2011;10(8):M110.006833.

6. Rienks M, Papageorgiou AP, Frangogiannis NG, et al. Myocardial extracellular matrix: an ever-changing and diverse entity. Circ Res. 2014;114(5):872-888.

7. Xia Y, Dobaczewski M, Gonzalez-Quesada C, et al. Endogenous thrombospondin 1 protects the pressure-overloaded myocardium by modulating fibroblast phenotype and matrix metabolism. Hypertension. 2011;58(5):902-911. 
8. Nishioka T, Onishi K, Shimojo N, et al. Tenascin-C may aggravate lef ventricular remodeling and function after myocardial infarction in mice. Am J Physiol Heart Circ Physio. 2010; 298(3):H1072-H1078.

9. Oka T, Xu J, Kaiser RA, et al. Genetic manipulation of periostin expression reveals a role in cardiac hypertrophy and ventricular remodeling. Circ Res. 2007;101(3):313-321.

10. Tian Y, Kelly-Spratt KS, Kemp CJ, et al. Mapping tissue-specific expression of extracellular proteins using systematic glycoproteomic analysis of different mouse tissues. J Proteome Res. 2010;9(11):58375847.

11. Tian Y, Koganti T, Yao Z, et al. Characterization of cardiac extracellular proteome and membrane topology using glycoproteomics. Proteomics Clin Appl. 2014;8(7-8):595-602.
12. Lee MC, Sun B. Glycopeptide capture for cell surface proteomics. J Vis Exp. 2014:9(87).

13. Collins BE, Paulson JC. Cell surface biology mediated by low affinity multivalent protein-glycan interactions. Curr Opin Chem Biol. 2004;8(6):617-625.

14. Zhang H, Liu AY, Loriaux $P$, et al. Mass spectrometric detection of tissue proteins in plasma. Mol Cell Proteomics. 2007;6(1):64-71.

15. Tian Y, Kelly-Spratt KS, Kemp CJ, et al. Identification of glycoproteins from mouse skin tumors and plasma. Clin Proteomics. 2008;4(3-4):117136

16. Schiess R, Wollscheid B, Aebersold R. Targeted proteomic strategy for clinical biomarker discovery. Mol Oncol. 2009;3(1):33-44. 